\title{
Alle Hebel zur Prognoseverbesserung ziehen
}

- Zu den Substanzen, mit denen sich die Mortalität von Herzinsuffizienzpatienten und die Häufigkeit ihrer Klinikaufenthalte vermindern lassen, gehören auch Mineralokortikoidrezeptor-Antagonisten (MRA).

Etwa 70\% der Patienten mit Herzinsuffizienz befinden sich im Stadium NYHA II, d. h. sie weisen eine milde Herzinsuffizienz auf. "Daran ist aber gar nichts milde außer den Symptomen, die der Patient hat", sagte Prof. Johann Bauersachs, Hannover. Die Mortalität beträgt bis zu 10\% pro Jahr. Mit jeder Klinikaufnahme steigt das Sterberisiko.

\section{Auch MR-Antagonisten berücksichtigen} Von den Medikamenten, die die Prognose verbessern, sind RAS-Blocker (ACE-Hemmer oder Angiotensin-Rezeptorblocker) und Betablocker gut etabliert. Mehr als $80 \%$ der Patienten werden damit behandelt. Doch maximal 40\% erhalten Mineralokortikoidrezeptor-(MR-)Antagonisten. „Wir müssen noch mehr dafür tun, dass auch diese hoch effektiven Substanzen häufiger im Therapieplan berücksichtigt werden", forderte Bauersachs.

Nach der kürzlich aktualisierten ESCLeitlinie (Eur Heart 2012; J 33: 1787-1847)

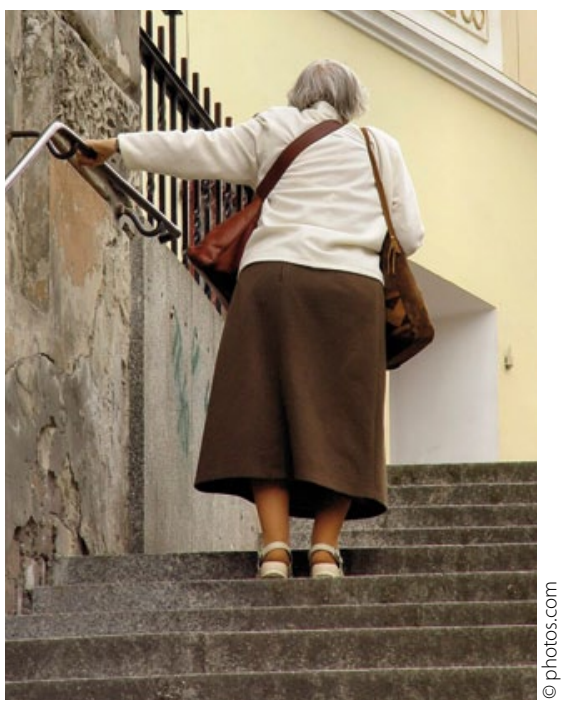

Auch eine milde Herzschwäche muss leitliniengerecht behandelt werden.

zur Behandlung der chronischen Herzinsuffizienz sollten alle Patienten, deren Symptome trotz Therapie mit RAS-Blockern und Betablockern persistieren (NYHA II-IV, Ejektionsfraktion $\leq 35 \%$ ), einen MR-Antagonisten erhalten, um das Risiko für Krankenhausaufnahmen und Mortalität zu vermindern (Empfehlung Klasse I, Level A).
Maßgeblich beeinflusst wurden diese Empfehlungen von den Ergebnissen der EMPHASIS-HF-Studie (New Engl J Med 2011; 364: 11-21), in der eine Therapie mit 25-50 mg Eplerenon (Inspra ${ }^{\oplus}$ ) pro Tag (zusätzlich zur medikamentösen Basistherapie) das Risiko für den primären Endpunkt (kardiovaskulärer Tod oder Herzinsuffizienz-bedingte Krankenhausaufnahme) gegenüber Placebo signifikant um 37\% ( $p<0,001)$, das Risiko für Hospitalisierung wegen Herzinsuffizienz um $42 \%(p<0,001)$ und die Gesamtmortalität um $24 \%$ $(p=0,008)$ gesenkt hat.

Nur für Eplerenon ist die Wirksamkeit bei leichter Herzinsuffizienz nachgewiesen. Man sollte sich davor hüten, die Ergebnisse auf Spironolacton zu übertragen, meinte Prof. Bertram Pitt, Ann Arbor/USA. Dem pflichtete auch Prof. Faiez Zannad Nancy/Frankreich bei: „25 mg Spironolacton wäre wahrscheinlich zu stark wirksam", sagte Zannad.

\footnotetext{
- Dr. Angelika Bischoff

Quelle: Symposium „Update your clinical practice based on the new 2012 ESC guidelines on the treatment of chronic heart failure", ESC-Kongress, München, August 2012 (Veranstalter: Pfizer)
}

\section{Zehn Jahre Olmesartan}

\section{Zulassungserweiterung zum Geburtstag}

- „Olmesartan ist das effektivste Sartan mit der geringsten Abbruchrate", berichtete Josep Redon, Valencia. Zum 10. Geburtstag konnte die Herstellerfirma bekanntgeben, dass die auf Olmesartan basierende fixe Dreierkombination Sevikar $\mathrm{HCT}^{\oplus}(\mathrm{Ol}-$ mesartan (OLM), Amlodipin (A) und Hydrochlorothiazid (HCT)) nun explizit auch in Deutschland für diejenigen Hypertoniker zugelassen ist, deren Blutdruck mit der Fixkombination Olmesartan/Amlodipin nicht ausreichend kontrolliert wird.

15-20\% der Patienten mit Hypertonie benötigen mehr als zwei unterschiedliche Antihypertensiva, um ihren Blutdruck auf die empfohlenen Zielwerte zu senken, be- tonte Redon. Durch die Indikationserweiterung für Sevikar $\mathrm{HCT}^{\oplus}$ ist es nun möglich, diese Patienten sofort auf eine fixe Dreifachkombination umzustellen, ohne dass zuerst noch freies HCT als zusätzliche Tablette gegeben werden muss.

\section{Einsparpotenzial: 1,8 Millionen Euro}

Es hat sich gezeigt, dass eine Fixkombination zur Bluthochdruck-Behandlung gegenüber einer Kombinationstherapie aus den jeweiligen Einzelmedikamenten aufgrund der geringeren Tablettenanzahl bei guter Verträglichkeit eine bessere Compliance gewährleistet. Außerdem ist diese Therapie kostengünstiger, wie Lorenzo Terranova,
Florenz, darlegte: Eine sozioökonomische Modellrechnung ergab, dass im Schnitt etwa 1,8 Millionen Euro eingespart werden könnten, wenn 10000 Hypertoniker von der freien Kombination zweier Antihypertensiva auf die Fixkombination derselben Komponenten umgestellt würden.

Als neuer, professionell entwickelter Service für Patienten wie auch für "Health Care Professionals" wird von DaiichiSankyo die Webseite www.hypertensioncare.eu angeboten (derzeit nur in Englisch).

- Dr. Jochen Aumiller

Quelle: Pressekonferenz "10 Jahre Olmesartan", Rom, Juni 2012 (Veranstalter: Daiichi-Sankyo) 